\title{
Vigilância nutricional e alimentar na infância e adolescência: retratos da realidade
}

\author{
Nutrition and food surveillance during childhood and adolescence: portraits of reality \\ Caroline Nicola Sangallia \\ a Nutricionista. Doutora em Ciências da Saúde.
}

\begin{abstract}
S egundo a Organização Mundial de Saúde, aproximadamente 42 milhões de crianças de até cinco anos $\checkmark$ de idade são classificadas com sobrepeso e/ou obesidade, das quais 31 milhões vivem em países em desenvolvimento ${ }^{1}$. No Brasil, dados da última Pesquisa de Orçamento Familiar (POF 2008/2009) mostraram prevalências de excesso de peso variando de 32 a 40\% entre as crianças de cinco e nove anos ${ }^{2}$. Estes resultados são alarmantes, uma vez que a infância é um período crítico para o ganho de peso excessivo, desenvolvimento da obesidade e suas doenças relacionadas ${ }^{3,4,5,6}$. Além disso, $60 \%$ dos obesos na primeira década de vida irão apresentar pelo menos uma alteração metabólica anos mais tarde 7 .

Nesse cenário, a literatura vem destacando modificações no consumo alimentar concomitantes com o aumento das prevalências de excesso de peso. Este consumo é caracterizado por alimentos com alta densidade de energia, excesso de gorduras totais e saturadas, e maiores concentrações de açúcar e sódio. Ao mesmo tempo, observa-se a diminuição no consumo de frutas, verduras e legumes ${ }^{8,9,10}$.

Nesta edição, três artigos evidenciam a importância da vigilância do estado nutricional em crianças e adolescentes, e destacam prevalências de excesso de peso que se aproximam dos dados nacionais, mesmo entre a população pediátrica em estado crítico. Por fim, um artigo trata a questão dos fatores associados ao consumo de frutas e verduras na adolescência. Cabe a nós, como profissionais da saúde, realizarmos estudos de vigilância nutricional, bem como investigações de fatores de risco modificáveis associados ao excesso de peso para subsidiar intervenções na tentativa de minimizar o aumento da epidemia da obesidade infantil.
\end{abstract}

\section{REFERÊNCIAS}

1. World Health Organization. Report of the commission on ending childhood obesity [Internet]. Geneva: WHO; 2016 [capturado 2018 dez. 2018]. Disponível em: http://apps.who.int/iris/ bitstream/10665/204176/1/9789241510066_eng.pdf

2. Instituto Brasileiro de Geografia e Estatística. Pesquisa de orçamentos familiares 2008-2009: antropometria e estado nutricional de crianças, adolescentes e adultos no brasil. Rio de Janeiro: IBGE; 2010.

3. Zheng M, Lamb KE, Grimes C, Laws R, Bolton K, Ong KK, Campbell K. Rapid weight gain during infancy and subsequent adiposity: a systematic review and meta-analysis of evidence. Obes Rev. 2018;19(3):321-32.

4. Bacha F, Gidding SS. Cardiac abnormalities in youth with obesity and type 2 diabetes. Curr Diab Rep. 2016;16(7):62.

5. Bass R, Eneli I. Severe childhood obesity: an under-recognised and growing health problem. Postgrad Med J. 2015;91(1081):639-45.
6. Li L, Pérez A, Wu LT, Ranjit N, Brown HS, Kelder SH. Cardiometabolic risk factors among severely obese children and adolescents in the United States, 1999-2012. Child Obes. 2016;12(1):12-19.

7. Srinivasan SR, Myers L, Berenson GS. Predictability of childhood adiposity and insulin for developing insulin resistance syndrome (syndrome X) in young adulthood: the Bogalusa Heart Study. Diabetes. 2002;51(1):204-9.

8. Bachman JL, Reedy J, Subar AF, Krebs-Smith SM. Sources of food group intakes among the US Population, 2001-2002. J Am Diet Assoc. 2008;108(5):804-14.

9. Levy-Costa RB, Sichieri R, Pontes NS, Monteiro CA. Household food availability in Brazil: distribution and trends (1974-2003). Rev Saúde Pública. 2005;39(4):530-40.

10. Piernas C, Popkin BM. Snacking increased among U.S. adults between 1977 and 2006. J Nutr. 2010;140(2):325-32. 